\title{
Les transitions démocratiques : mobilisations collectives et fluidité politique
}

\section{Richard Banegas}

\section{(2) OpenEdition \\ 1 Journals}

\section{Édition électronique}

URL : http://journals.openedition.org/conflits/443

DOI : $10.4000 /$ conflits.443

ISSN : $1777-5345$

Éditeur :

CCLS - Centre d'études sur les conflits lilberté et sécurité, L'Harmattan

Édition imprimée

Date de publication : 15 décembre 1993

ISSN : 1157-996X

Référence électronique

Richard Banegas, «Les transitions démocratiques : mobilisations collectives et fluidité politique », Cultures \& Conflits [En ligne], 12 | hiver 1993, mis en ligne le 14 mars 2006, consulté le 30 mars 2021. URL : http://journals.openedition.org/conflits/443 ; DOI : https://doi.org/10.4000/conflits.443

Ce document a été généré automatiquement le 30 mars 2021.

Creative Commons License 


\title{
Les transitions démocratiques : mobilisations collectives et fluidité politique
}

\author{
Richard Banegas
}

Repenser l'incertitude des transitions

2 Comment penser l'incertitude fondamentale des "transitions démocratiques"1 ${ }^{\text {? Peut- }}$ on, au delà de leur chaos apparent, discerner des logiques propres à ces situations qui nous éclairent sur le "comment" des processsus de démocratisation et non plus seulement sur leur "pourquoi"? Peut-on découvrir des propriétés contextuelles qui nous aident à comprendre les problèmes de gestion des réformes, de légitimation, de consolidation démocratique, ou les phénomènes de mobilisation collective qui marquent ces périodes de flux, de changement.

3 Les modèles et approches "classiques" de la démocratisation ne semblent pouvoir en rendre compte, faut-il alors adopter une position alternative dérivée de l'analyse des crises politiques (fluidité) et des mobilisations collectives? Ce sera notre hypothèse. La "transitologie" (P. Schmitter) a produit une abondante littérature, dont les inflexions théoriques ne sont pas sans rapports avec les détours de l'"Histoire réelle" et de la construction démocratique. Grossièrement, au temps long des premières vagues semblent correspondre les analyses structurelles, fondées sur des macro-variables d'ordre culturel, économique ou social (religion, culture civique, structure agraire, niveau de richesse économique...). Citons pour mémoire les travaux d'Almond et Verba sur la culture civique, ceux de D. Lerner, S. Eisenstadt sur la modernisation, ceux de B. Moore ou A. Gerschenkron (sur les rapports pluralisme/révolution agro-commerciale ou révolution industrielle) et surtout la thèse, controversée, de S.M. Lipset sur les préconditions socio-économiques de la démocratie ${ }^{2}$. Ces approches macro-historiques ${ }^{3}$ ont contribué à l'intelligence des conditions d'émergence, de consolidation et de 
stabilité des régimes pluralistes. Mais elles ont aussi subi la critique pour leur biais déterministe, parfois finaliste et surtout pour leur tendance à confondre corrélations statistiques avec causalité historique. Si des conditions culturelles ou économiques paraissent préserver la stabilité, le fonctionnement d'une démocratie, peut-on assurer qu'elles sont nécessaires à son avènement ? La perspective, remarque D. Rustow ${ }^{4}$, est souvent plus "fonctionnelle" que "génétique" et néglige la dimension politique. Au mieux, elle nous instruit sur les probabilités de réusssite des démocratisations et sur le "pourquoi" des transitions, jamais sur le "comment".

Ces impasses, combinées à l'accélération des changements de la "Troisième vague" 5 , au rôle clé de certains acteurs des transitions (Suarez, Caramanlis...) ont conduit à des réorientations théoriques: l'action des leaders fut réévaluée et l'attention s'est déplacée vers les facteurs de court terme, les variables micropolitiques. Dès la fin des années 1970, le paradigme du choix rationnel, de l'interaction stratégique s'est imposé pour rendre compte des processus de transition eux-mêmes et non plus seulement de leurs conditions d'émergence ${ }^{6}$. Deux propositions fondent cette réorientation : d'abord, l'idée que les variables politiques importent plus que les autres dans la compréhension des processus de transition. Ainsi "l'ouverture", la libéralisation, des régimes autoritaires ne pourrait se comprendre qu'au regard de la lutte pour le pouvoir que se livrent les divers groupes et factions au sein de l'Etat ${ }^{7}$. Ensuite le postulat selon lequel les transitions démocratiques sont des situations d'anormalité où l'action stratégique des élites, le talent, l'habileté politique des leaders sont décisifs pour penser le "moment" du changement de régime et comprendre les divergences de trajectoire. Cette réhabilitation de l'action, des variables politiques a aussi permis d'insister de façon salutaire sur le caractère indéterminé des transitions démocratiques. L'incertitude est désormais introduite comme "paramètre" central des transitions. C'est elle qu'il faut saisir pour comprendre les processus de démocratisation dans leur complexité. O'Donnell et Schmitter invitent à la prendre comme objet d'étude, A. Przeworski en fait l'élément central de sa théorie (la démocratisation comme

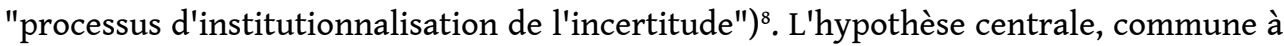
l'ensemble de ces analyses centrées sur la dimension politique et l'incertitude est la suivante : les origines et l'évolution des régimes démocratiques sont déterminées moins par les facteurs culturels ou économiques que par les actions, les choix des élites clés qui cherchent à maximiser leurs intérêts dans un cadre institutionnel en flux que leur lutte contribue à façonner. A l'intérieur de ce paradigme du choix rationnel, l'accent est mis sur diverses variables politiques : nature du leadership, du régime autoritaire (ou totalitaire); composition et division du bloc au pouvoir ; performance/efficacité/ légitimité du gouvernement; autonomie/contrôle des forces armées; soutiens/ oppositions dans la "société civile"... Deux modèles célèbres peuvent illustrer ces réorientations théoriques. Celui de $\mathrm{D}$. Rustow, d'abord, qui à partir d'une critique des "approches fonctionnelles" (préconditions) cherche dès 1970 à construire un modèle politique "génétique" des transitions. Le processus commence en général par une "phase préparatoire" marquée d'un "conflit politique prolongé et insoluble" qui force les protagonistes à engager des réformes de libéralisation. "La démocratie n'est pas l'objectif premier, originel; elle est recherchée pour d'autres fins, elle émerge comme un sous-produit fortuit du conflit, de la lutte"9. Cette phase préparatoire se clôt par la décision consciente des élites de s'entendre sur des règles minimales, d'institutionnaliser le pluralisme pour sortir de l'impasse. La phase de consolidation, qui suit la concrétisation de ce compromis est une période de sélection des acteurs et 
d'apprentissage-acceptation des règles ("habituation phase") ${ }^{10}$ Rustow insiste donc sur la dimension conflictuelle des transitions, sur les choix politiques cruciaux qui sont effectués par les élites dans ce contexte de polarisation, d'incertitude et met l'accent sur la dynamique du processus lui-même. Juan Linz ${ }^{11}$, de la même manière, affirme l'importance, "surdéterminante" des variables politiques. Dans les phases de changement, de rééquilibrage, le choix des élites est crucial; la formulation de "l'agenda initial", son habile gestion, fixe dès le départ le sort des transitions. Il s'agit pour les nouveaux dirigeants de faire la preuve de leur capacité à gouverner et affirmer ainsi la légitimité du nouveau régime. Au coeur de ce "programme des urgences de la légitimation démocratique" (G. Hermet) se joue la gestion des soutiens et des oppositions ("loyales/semi-loyales/déloyales") et la formation des prédispositions de base envers le nouveau régime. En désignant ainsi les impératifs d'une démocratisation réussie, Juan Linz souligne donc l'importance stratégique de l'action des élites politiques. Il ouvre la voie à l'analyse des transitions en termes d'interaction stratégique mais aussi aux "explications" volontaristes du changement.

Ces dernières se fondent sur le postulat que "la démocratisation est essentiellement une affaire d'habileté politique"12, de bricolage minutieux. Elles oublient que la démocratie émerge la plupart du temps comme un choix par défaut ("second best choice"), une solution contingente à un conflit insoluble ${ }^{13}$, que "les meilleurs stratèges de la démocratisation ne sont pas toujours les démocrates les plus convaincus" ${ }^{14}$. Elles imputent aux acteurs une capacité de contrôle là où souvent nécessité fait loi, méconnaissent la complexité des processus de décision et négligent les contraintes stucturelles. Surtout, par son indifférence à l'état conjoncturel des structures sociales en période de fluidité , "l'illusion héroïque" (M. Dobry) passe à coté des ressorts profonds des processus de transition. La réévaluation des variables politiques a aussi mené à des formalisations plus ou moins poussées des choix et des interactions stratégiques des acteurs clés des transitions. L'accent y est mis sur les problèmes de perception de la réalité dans les situations d'incertitude où l'évaluation des conditions et des ressources est difficile à faire. D'où le succès de ces travaux qui semblaient fournir les clés pour penser les processus de transition en tant que tels, dans leur complexité et leur indétermination. Trois modèles peuvent illustrer ces approches "stratégiques": celui d'A. Hirschman ("reform mongering"), celui des seuils de transition d'A. Przeworski et celui de la consolidation démocratique de G. O'Donnell ${ }^{15}$. Hirschman se demande comment, dans le contexte d'incertitude des transitions, les gouvernants peuvent accroître la probabilité de succès des réformes par la construction de coalitions politiques. Przeworski analyse les probabilités et modalités de chute des régimes autoritaires en fonction des risques (et des changements de perception des risques) à l'approche des seuils de transition, tandis qu'O'Donnell examine les diverses stratégies qui s'offrent aux acteurs pour consolider la démocratie, prévenir le retour à l'autoritarisme ${ }^{16}$. Les trois modèles visent, chacun selon des critères différents, à identifier les acteurs clés, à formaliser leurs objectifs, la distribution de leurs préférences pour déduire de ces paramètres et de la structure du "jeu" les "solutions d'équilibre" susceptibles d'émerger. La décision d'ouverture des régimes dictatoriaux, la dynamique de libéralisation-démocratisation sont ainsi expliquées rationnellement par des dynamiques de convergence, la démocratie vue comme un simple état d'équilibre entre stratégies conflictuelles. Malgré leur formalisme, ces théories sont d'un intérêt certain. En insistant sur les problèmes de perception, d'évaluation (des préférences, des ressources, des "coups" de l'adversaire) 
en situation d'incertitude, elles touchent au coeur de la dimension stratégique des transitions. L'accent est mis par exemple sur la perception des probabilités de succès ou la variation des coûts et des bénéfices au cours du jeu. La perspective dynamique de l'interaction est importante. Elle permet de comprendre la façon dont les parties révisent leurs attentes et leurs objectifs dans le conflit. Sur ce point, on peut cependant regretter que les divers modèles intègrent peu les apports des théories des conflits, notamment de Schelling ${ }^{17}$. Ils souffrent aussi d'autres limites. Hormis leur caractère anhistorique et simplificateur (en général deux parties ou quatre acteurs) ils semblent conduire à des impasses : ainsi Przeworski, après avoir testé plusieurs hypothèses tend à conclure à une impossibilité logique de la démocratisation. Il doit alors sortir de la logique formelle de la théorie des jeux pour trouver les raisons de l'ouverture, de la libéralisation (explication psychologique) ${ }^{18}$. En outre, ces modèles s'en tiennent à des jeux où les préférences sont fixées (par l'analyste). Or le propre des situations de transition, de fluidité politique, n'est-il pas justement la mobilité, la plasticité des préférences et des perceptions? Cette hypothèse nous semble centrale pour la compréhension des processus de démocratisation. Enfin, ces approches stratégiques négligent les données contextuelles et structurelles des transitions.

6 Est-ce à dire qu'entre les analyses structurelles et stratégiques, il suffit d'adopter une posture médiane pour comprendre les processus de transition dans leur dynamique et leur complexité ? Afin d'échapper à l'alternative stérile "déterminisme des structures / liberté de l'acteur", T.L. Karl et P. Schmitter proposent par exemple de passer de l'étude $\mathrm{du}$ "choix contingent à la contingence structurelle"19, d'arrêter "la recherche futile de préconditions" sans pour autant négliger "les limites et les chances que les structures sociopolitiques solidement ancrées peuvent assigner à un choix contingent". Elles constituent des "conditions limitatives" qui, dans ces situations d'incertitude, pèsent sur le choix des acteurs. L'argument n'est pas neuf, on retrouve les "confining conditions" d'O. Kirchheimer ou le "modèle probabiliste" de J. Linz. D'autres approches visent à dépasser ce dilemme: celle "possibiliste" d'Hirschman où les conditions défavorables peuvent se muer en "bénédictions déguisées", où les actions peuvent avoir des "conséquences inattendues". Celle des "conjonctures critiques" proposée par Ruth et David Collier où les choix politiques répondent d'une part à des clivages sociaux profonds et d'autre part contribuent eux-même à façonner sur le long terme l'arène politique, les contraintes futures (ici ce sont les choix, les modalités d'incorporation de la classe ouvrière qui, adoptés dans des conjonctures critiques, configurent les régimes latino-américains) ${ }^{20}$.

Or il nous semble que ces stratégies, ces interactions qui "façonnent le cadre institutionnel en flux" doivent s'interpréter non seulement en fonction des opportunités et contraintes préexistantes mais aussi et surtout au regard de celles qui naissent de la transition elle-même. Prendre en compte l'héritage ne suffit pas. Ne fautil pas aussi s'attacher aux transformations que subissent les "structures" dans ces périodes critiques (sous l'effet notamment des mobilisations collectives), s'intéresser à l'état conjoncturel des configurations sociales et analyser le comportement tactique des acteurs dans ce cadre, dans ce contexte de fluidité ? Pour penser l'incertitude des démocratisations, ne faut-il pas, d'une part chercher leurs origines dans les propriétés du contexte d'action "transition" et d'autre part rapporter les problèmes stratégiques de gestion et de consolidation démocratique aux propriétés contextuelles de la fluidité politique? S'intéresser ainsi à l'état conjoncturel des rapports sociaux serait une autre 
façon de saisir le lien qui s'établit dans ces moments critiques entre le macrosocial et le micropolitique.

\section{Les transitions comme situations de fluidité politique}

Il s'agit alors de considérer les transitions comme des situations de crise qui affectent l'ensemble des rapports sociopolitiques, de prendre en considération ce contexte de crise dans sa double dimension conjoncturelle et structurelle et voir "ce qui se joue" dans le processus même de crise, de transition. Ne peut-on discerner des logiques, des traits propres à ces conjonctures critiques, qui nous aident à penser l'incertitude des processus de démocratisation? Pour chercher ces "règles qui gouvernent le chaos" ${ }^{21}$, il nous semble intéressant de prolonger la thèse de M. Dobry et d'appréhender les transitions comme des situations de fluidité politique, de "crise multisectorielle"22 qui se caractérisent au niveau "structurel" par un trait central : le bouleversement, sous l'effet des "mobilisations multisectorielles", des rapports habituellement en vigueur entre groupes sociaux (la "désectorisation conjoncturelle de l'espace social"). "Désectorisation" qui induit d'autres propriétés ( "crise des transactions collusives", désobjectivation des rapports sociaux...) et définit, au niveau micropolitique un contexte d'action original qui pèse sur les calculs, les anticipations, les stratégies des acteurs, la valeur des ressources et leur efficacité ("contexte d'interdépendance tactique élargie"). Penser les transitions, comme des situations de fluidité politique, c'est partir d'une double hypothèse: d'une part l'incertitude "structurelle" des transitions dérive de ces propriétés, est liée à un état particulier des "structures", des rapports sociaux, qui subissent sous l'effet des mobilisations de profondes transformations conjoncturelles. D'autre part, les problèmes qui se posent aux acteurs dans la gouvernabilité, la gestion à court terme des transitions (perspective linzienne) tout comme à moyen terme les modalités de légitimation et de consolidation démocratique sont à rapporter à ces propriétés contextuelles de la fluidité politique. Adopter cette posture alternative pour analyser "ce qui se joue" dans des transitions, c'est donc poursuivre un double programme de recherche: caractériser d'abord la fluidité politique des transitions, mettre à jour les propriétés macro et microsociologiques pour mieux comprendre ensuite les problèmes stratégiques et tactiques des divers acteurs.

Pour bâtir sa théorie des conjonctures fluides, M. Dobry s'appuie d'abord sur une critique du modèle complexe d'Almond et Flanagan qui abordent deux phénomènes importants pour notre propos: la dynamique des processus de crise et la fluctuation parallèle de la valeur des ressources ${ }^{23}$. Leurs intuitions selon M. Dobry pourraient se ramener à ceci: la "valeur" des ressources politiques connait de très sensibles variations dans les conjonctures critiques et ces fluctuations seraient en rapport avec la dimension "multisectorielle" des mobilisations collectives. Trois "arènes" sont distinguées : celle où jouent les ressources coercitives, celle où se joue l'influence et celle où comptent les ressources institutionnelles. La valeur de celles-ci fluctuerait corrélativement au poids de chaque arène, variable selon les séquences de la crise. Malgré leur intuition, remarque M. Dobry, les deux auteurs ne perçoivent pas que sous l'effet des mobilisations multisectorielles, ce sont les rapports entre arènes, entre champs sociaux qui sont transformés et que la variation pondérale des ressources est 
liée à ce phénomène. Ils sont indifférents à l'état conjoncturel des configurations sociales. Les chercheurs de Stanford placent cette réflexion dans la dynamique des processus de crise que Flanagan décrit comme le passage d'un "système à monnaie dominante" à un "système à monnaies multiples" où la coercition servirait d'étalon de mesure ultime aux autres ressources. La perspective est intéressante pour rendre compte de la dynamique des transitions où l'on observe cette fluctuation de valeur des ressources et l'importance centrale du monopole de la violence. On peut toutefois remarquer avec M. Dobry que les conjonctures les plus fluides ne sont pas les situations où l'on assiste à un recours généralisé à la coercition (qui devient monnaie dominante), mais les "situations incertaines où différentes ressources, relativement cloisonnées les unes par rapport aux autres dans les conjonctures routinières viennent à être confrontées du fait des mobilisations et de la transformation corrélative des rapports entre les sites sociaux où ces ressources opèrent"24. La crise vénézuélienne de 1958 en fournit l'illustration. Les mobilisations multisectorielles et la dynamique du changement font naître un "système à monnaies multiples" où la valeur des ressources subit de rapides et fortes fluctuations. Dès la fin de 1957, avec la crise de succession du général Pérez Jiménez, on observe en effet un déplacement du site de décision et $\mathrm{d}$ 'action politique, de l'arène institutionnelle à l'arène où dominent les jeux d'influence (discussions au sein de l'armée entre les factions rivales pour décider du sort du général-président et trouver une issue à la crise ministérielle). Puis de l'arène où les forces armées dominent (deux tentatives de coup d'Etat) à la rue où priment les ressources de mobilisation. Une dévaluation des diverses "monnaies" s'opère alors au cours du conflit: une fois épuisées les ressources institutionnelles (Pérez Jiménez cherche, face aux échéances électorales qui approchent, à se maintenir au pouvoir par des moyens institutionnels légaux, par l'organisation d'un plébiscite), les acteurs dominants expérimentent d'autres moyens (truquage des élections, manipulation du plébiscite) qui sont à leur tour dévalorisés par la réaction des adversaires : les partis, les groupes d'opposition usent de leurs propres "liquidités" (l'action collective, les grèves, les manifestes...) pour imposer un changement de régime, tandis que les diverses tendances de l'armée cherchent à imposer par la force leur solution, à imposer la violence comme "étalon de mesure" des autres ressources. Cependant ce processus de passage d'une arène à l'autre, de dévaluation des "monnaies", n'est ni linéaire ni progressif. Le poids des ressources augmente ou diminue selon les moments : ainsi le jeu des influences après avoir laissé place en décembre 1957 à la violence reprend de la valeur fin janvier lorsque la nouvelle junte militaire tente de coopter des membres de l'opposition. Les variations, les fluctuations conjoncturelles qui s'observent dans la dynamique des processus de crise, de transition, dérivent des transformations d'état que subissent les configurations sociales sous l'effet des "mobilisations multisectorielles" et en premier lieu de la transformation conjoncturelle des rapports entres secteurs ou champs sociaux.

11 La "désectorisation conjoncturelle de l'espace social" si l'on suit M. Dobry, est la propriété centrale des conjonctures fluides. Riche en implications théoriques et empiriques, elle peut aider à penser l'incertitude des transitions dans sa dimension à la fois structurelle et conjoncturelle. Elle se comprend par contraste, avec les situations normales des sociétés complexes ${ }^{25}$ où le jeu est "sectorisé", c'est à dire où les divers secteurs sociaux sont à la fois dans des relations d'autonomie et d'interdépendance, où leurs "logiques" s'imposent de façon routinière aux acteurs; où l'objectivation des rapports sociaux et la stabilité sociale sont assurées notamment par des 
reconnaissances réciproques, des réseaux d'interaction consolidés entre secteurs (les "transactions collusives intersectorielles" pour reprendre les termes de M. Dobry). La "désectorisation conjoncturelle de l'espace social" désigne les "transformations d'agencement des secteurs les uns par rapport aux autres"26, les bouleversements que subissent ces rapports sous l'effet des mobilisations multisectorielles. C'est la remise en cause des configurations sociales stabilisées et objectivées par les "transactions collusives". M. Dobry distingue trois dimensions de cette crise des rapports sectoriels, importantes pour l'étude des transitions. La perte d'autonomie des secteurs sociaux d'abord. Elle est dûe au caractère même des mobilisations de crise, c'est à dire "aux décloisonnements, aux interférences des logiques sectorielles et à l'occurrence de coups trans-sectoriels" 27 dont ces mobilisations sont porteuses. Elle s'observe au niveau des rapports inter-sectoriels : le champ politique, en temps de crise, semble en effet perdre de son autonomie, être plus perméable, plus sensible aux demandes, aux coups des acteurs "extérieurs". Elle s'observe aussi et surtout au niveau de chaque secteur social où l'emprise des "logiques" de chacun tend à décliner avec la "désectorisation". La réduction de l'emprise des "logiques" sectorielles se manifeste par le fait que les acteurs, situés dans un champ affecté par des mobilisations multisectorielles tendent à utiliser pour leurs calculs, leurs anticipations, un univers de référence, des critères et des repères d'évaluation largement externes à la logique propre à leur secteur ${ }^{28}$. Cette "évasion des calculs", cette baisse d'endo-référence des champs sociaux se traduit par l'affaiblissement des critères et repères routiniers. La mobilité accrue des enjeux est corrélative à cette désectorisation de l'espace social. Avec le "désenclavement des arènes" (M. Dobry), les enjeux perdent leurs spécificités sectorielles, ils deviennent centraux pour d'autres groupes sociaux, d'autres acteurs les investissent de sens, en font les enjeux de leur propre mobilisation. Cette propriété est essentielle pour comprendre les dynamiques de l'action collective en période de transition. Ainsi par exemple, en 1980-81, le statut des ouvriers de Gdansk se "dé-spécifie" en se politisant. Il est investi de sens par d'autres acteurs qui l'utilisent pour se mobiliser, prend une dimension nationale et une charge idéologique forte. Dans la dynamique des mobilisations multisectorielles, cet enjeu déborde le cadre restreint des ouvriers de Gdansk (on passe de la revendication du statut d'ouvrier à la revendication d'un statut officiel pour Solidarité), puis il déborde le champ syndical avec l'intervention du secteur religieux, des intellectuels dissidents, pour devenir un enjeu politique, institutionnel, qui va nourrir la contestation pendant les années 1980 et conduire à la Table Ronde. Cette mobilité des enjeux s'observe dans la plupart des cas. Au Bénin, lorsque les étudiants se mettent en grève en janvier 1989, occupent le campus d'Abomey-Calavi, c'est d'abord pour réclamer le versement des arriérés de bourse. Les enseignants, les fonctionnaires et une partie de l'armée les rejoignent dans la protestation sur le même thème des arriérés de salaires. La mobilisation devient multisectorielle et une dynamique de "désectorisation" s'enclenche. Rapidement les revendications et les enjeux matériels se chargent d'un nouveau sens, sont investis par d'autres acteurs (notamment les évêques, Mgr De Souza en tête, qui diffusent alors une lettre pastorale appellant au changement de régime). Autre témoin de cette fluidité et de cette "récupération" des enjeux, la "Charte des Droits de l'Homme et de l'Etudiant" qu'appellent de leurs voeux certains groupes et syndicats d'opposition camerounais.

D'autres propriétés sont liées à cette destructuration conjoncturelle de l'espace sociopolitique. La "désobjectivation des rapports sectoriels" ${ }^{29} \mathrm{n}$ 'est pas la moindre. Elle représente une des vulnérabilités majeures des régimes politiques en situations de 
crise, de transition. Avec la "désectorisation", l'effondrement des relations habituelles entre secteurs ("crise des transactions collusives"), c'est l'objectivation des rapports sociaux, la légitimité de l'ordre sociopolitique qui est remise en cause. Remise en cause des accords et des reconnaissances entre les divers champs et groupes sociaux, érosion de cette forme de "complicité" qui en temps ordinaire assure le bon fonctionnement des régimes et les pratiques sociales légitimes. S'efface alors la représentation objectivée des rôles, des positions, des institutions et des domaines d'activité spécialisés qui fondaient une certaine réalité sociale. La crise béninoise de 1989-90 l'illustre à nouveau. Dans l'action collective, la dynamique de "désectorisation", de rapides désobjectivations s'opèrent. "Ceux qui sont engagés dans les grèves et les manifestations, témoigne un observateur, entreprennent un processus d'apprentissage politique" ${ }^{30}$, de prise de conscience, qui les mène, selon les mots d'une opposante, "de la lutte pour les salaires à la lutte morale". Ils ne font pas que contester la légitimité du régime, ils font aussi l'expérience d'une alternative. En 1989, les différentes structures officielles d'encadrement sont de plus en plus désertées et surtout contestées lorsque les grèves éclatent et que les leaders syndicaux, cooptés, s'avèrent freiner la contestation. Se créent alors des organisations parallèles qui, à partir de juin 1989, se dotent de structures de coordination. Des réseaux se mettent en place entre les différents lieux d'occupation et de contestation, un maillage territorial, se constitue peu à peu. C. Allen estime que ce mouvement d'auto-organisation fut un élément majeur du processus de politisation, de prise de conscience : "les grévistes y ont vu une leçon objective (prouvant) la nécessité de réformer le système politique dans son entier et la valeur du pluralisme institutionnel" ${ }^{13}$. Les témoignages semblent confirmer l'importance de ce processus de désobjectivation. Le même phénomène est rapporté par J. Staniszkis qui analyse la situation polonaise. Dès 1980, "les travailleurs, en s'emparant de la chose publique, ont réussi à vaincre la réification de l'ordre hiérarchique" ${ }^{32}$. Certes le processus de désobjectivation a été préparé par l'évolution des formes d'opposition depuis 1976 (KOR), mais plusieurs facteurs le précipitent. Les grèves d'août 1980 d'abord, qui prouvent qu'un refus d'obéissance peut paralyser un régime apparemment inaltérable et qui offrent, avec l'organisation du mouvement ouvrier, l'expérience d'une alternative. Malgré son échec, cela constitue "une première leçon de ce qu'est la légitimité" ${ }^{33}$. Le développement et l'institutionnalisation de Solidarité ensuite participent à ce mouvement de désobjectivation, de déconstruction sociale de la réalité. Staniszkis montre cependant l'aspect conjoncturel du phénomène : la dé-réification reflue avec l'affaiblissement de la mobilisation et la "normalisation" du régime (le terme de normalisation est d'ailleurs significatif : au delà de la répression, ce qui est en jeu c'est aussi et surtout la voûte de légitimation du régime ${ }^{34}$ ).

13 Ces déperditions conjoncturelles d'objectivation, convient M. Dobry, ne sont pas facile à mesurer. Elles s'observent par exemple dans les sentiments de folie, de libération, de fête qui marquent les crises mais aussi les transitions démocratiques. Dans ces moments, l'objectivation des divisions sociales, qui se traduit, normalement par une certaine impersonnalité des rapports entre rôles institués, semble affectée. En témoigne, l'occurrence des rapports de face à face qui rompent avec les arrangements institutionnels normaux, les mouvements de "fraternisation" (entre les manifestants et les policiers lors de la chute du Mur de Berlin, entre les comédiens et les ouvriers à Prague dans le théatre de la Lanterne Magique ${ }^{35}$ ) qui expriment une transgression des distances sociales objectivées, qui traduisent au fond la désectorisation conjoncturelle de l'espace social. 
14 L'incertitude structurelle des conjonctures fluides, des transitions en découle directement. Effet direct de la désectorisation, du décloisonnement des logiques sectorielles, de la désobjectivation, elle prend son origine dans les transformations d'état des rapports sociaux. Elle a une dimension psychologique évidente, mais elle est d'abord relationnelle, "structurelle"136.

$15 \mathrm{Au}$ niveau micropolitique, la transformation des rapports intersectoriels induit également des propriétés importantes pour la compréhension des processus de transition. L'identité de tout individu est plurielle, c'est une évidence. Liée à la multipositionnalité des acteurs dans les divers secteurs sociaux, elle se forge à travers la multiplicité des rôles, des registres qui s'offrent à eux. On perçoit alors l'effet que peut avoir "une dynamique sociale qui affecte précisément ce que l'on pourrait appeler le support social de la multidimensionnalité de l'identité personnelle": les mobilisations multisectorielles, la désectorisation de l'espace social "tendent à réduire l'identité à une dimension unique servant d'indice pratique dans les interactions". Une qualité, et souvent une seule, est sélectionnée comme indice pratique et constitue un "opérateur d'identification à vocation universelle (...) c'est à dire qui tend à être efficace dans l'ensemble de l'espace social" ${ }^{37}$. Que ce soit la qualité "d'aristocrate", de "travailleur", de "patriote", elles correspondent chacune à une "simplification" des identités, à des stigmatisations qui jouent un rôle crucial dans les luttes politiques et les mobilisations collectives. Cette tendance à la simplification des identités, liée au changement d'état des rapports sociaux est intéressante pour la compréhension des transitions. Les qualités de "communiste", de "fasciste" de "dissident" ou "d'opposant" s'imposent dans la lutte et tendent à simplifier la représentation du conflit entre "hardliners" et "softliners", entre "Eux" et "Nous" pour reprendre l'expression polonaise. Bien plus, la dynamique "d'unidimensionnalisation" tend à imposer une identité comme seule valable et légitime: la qualité de "démocrate". Ressource politique essentielle pour l'accès au pouvoir ou la reconversion ${ }^{38}$, c'est l'affirmation identitaire la plus répandue des temps de transition. Dans son modèle, D. Rustow soulignait déjà ce phénomène en parlant du processus de "sélection darwinienne" qui, entre les partis et les leaders politiques, arbitrait en faveur des démocrates. Il faut toutefois souligner le caractère temporaire de cette "unidimensionnalisation". La consolidation démocratique (cristallisation et institutionnalisation d'un système pluraliste de valeurs) ou son enlisement et son cortège de désenchantements ${ }^{39}$ s'accompagnent en général d'une redéfinition et une multiplication des pôles d'identification. La prolifération des nationalismes dans les années 1990 est là pour en témoigner. Avec la tendance à l'unidimensionnalisation des identités, apparaît déjà le lien qui s'établit, en période de transition, entre les transformations macrosociologiques (désectorisation...) et les comportements micropolitiques. Il apparaît encore plus clairement dans une propriété, centrale, touchant directement au comportement tactique des acteurs.

16 L'émergence d'un contexte d'interdépendance tactique élargie, le passage d'une forme d'interdépendance sectorielle à une forme élargie d'interdépendance est, au plan micropolitique, la principale propriété qui dérive de la dynamique des mobilisations et de la transformation des rapports entre secteurs sociaux. Elle influe directement sur la conduite des acteurs: ce sont les propriétés de ces "contextes d'interdépendance élargie" qui définissent les contraintes, les logiques d'action propres aux conjonctures fluides. Celles-ci se retrouvent dans les contextes de transition. "Avec la désectorisation 
de l'espace social et le désenclavement des sites de confrontation, affirme M. Dobry, on assiste à l'émergence d'une forme élargie d'interdépendance qui se substitue aux formes d'interdépendance plus locales, cloisonnées et fortement marquées par les logiques sectorielles" ${ }^{14}$. Autrement dit, il y a dans les conjonctures fluides, une tendance à l'interdépendance croissante des tactiques d'acteurs localisés en des sites différents de l'espace social. C'est à dire passage d'une forme routinisée d'interdépendance à l'intérieur d'un secteur particulier à une forme d'interdépendance élargie qui "tend à confronter directement des lignes d'action et des ressources jusque là cloisonnées et à déterminer leur valeur, leur efficacité dans cette confrontation" ${ }^{41}$. La perspective est intéressante pour l'étude des transitions où l'on observe ces phénomènes de confrontation directe entre acteurs jusque là isolés, prisonniers de leurs "logiques sectorielles" ou, plus prosaïquement, sommés par le pouvoir de s'y conformer (si l'on songe aux intellectuels dissidents). Point n'est besoin d'entrer dans le détail de cette forme émergente d'interdépendance "trans-sectorielle" (prolongeant E. Goffman, M. Dobry la caractérise par le "relâchement du lien entre un coup et son résultat", comme un "jeu tendu imparfait"). Il suffit, pour notre propos, de relever les effets de ce contexte sur le comportement des acteurs. D'abord, l'efficacité des coups n'est pas équivalente à leur "contenu" : il y a souvent une disproportion entre "petits coups" et "grands effets" que les acteurs ont du mal à maîtriser. Dans ce contexte, l'efficacité des coups ainsi que la "valeur" des ressources sont variables, liées à la dynamique de l'interaction, de la confrontation "trans-sectorielle". De fait, ce "relachement" du lien coup/résultat tend à réduire le contrôle qu'ont les individus sur la portée de leurs actes et sur la signification qui leur est attachée au cours du conflit. Il pèse aussi sur la crédibilité des actes, des messages, des menaces ou des promesses. Enfin on peut, avec M. Dobry, avancer l'hypothèse que "dans de telles conjonctures, où les acteurs tendent à être privés des points de repère (...) liés aux logiques sectorielles, leurs perceptions, anticipations et calculs ne peuvent contourner la dimension physique, la matérialité de ces échanges de coups et de leurs résultats locaux" ${ }^{42}$. Ceux-ci agissent comme des points attracteurs, des "saillances situationnelles" qui, dans le conflit, marquent la lutte pour l'interprétation, la définition des situations. Cette importance conjoncturelle des "saillances", des "points focaux" (Schelling) est un élément central des conjonctures fluides.

On perçoit sans peine, à travers ces propriétés, les traits propres aux contextes d'action stratégique des transitions. Le jeu des "saillances", les problèmes de maîtrise des coups, d'évaluation des ressources, de crédibilité, s'observent en effet dans le domaine central de la gestion des "agendas", mais aussi dans les compétitions symboliques pour la définition de la réalité, dans les luttes d'affirmation identitaire et dans la dynamique même des transitions et des mobilisations. L'importance des affirmations identitaires, des stigmatisations dans les contextes critiques a déjà été soulignée. il faut préciser comment elles s'opèrent concrètement pour saisir les effets de la fluidité politique, du "contexte d'interdépendance élargie" sur le comportement stratégique et tactique des acteurs. L'affirmation d'identité, la "labélisation" (H. Becker), comme on l'a vu avec le phénomène de "simplification" identitaire, se fait par la sélection et la production de sens de discours concurrents. (C'est là un des rôles de l'idéologie dans la mobilisation sur lequel il est inutile d'insister). Mais du fait des propriétés de l'interdépendance "élargie" (rôle des coups directs...), ce marquage se fait aussi et surtout, dans les conjonctures fluides de transition, par des actes concrets de positionnement, des coups tactiques qui sont autant d'affirmations identitaires. Du côté des gouvernants, ce peut 
être l'utilisation de la force armée, la mise en place de négociations, la définition ou la promesse d'un agenda de réformes..., du côté des oppositions, le déclenchement d'une grève, la diffusion de tracts, la participation à des manifestations, des défilés. On perçoit avec les récents travaux sur l'action collective (d'A. Pizzorno, et d'A. Melucci notamment) la dimension identitaire de ces mouvements de mobilisation: l'engagement individuel n'est pas uniquement un coût, c'est aussi une gratification, une affirmation d'identitét ${ }^{43}$. Cette fonction de marquage par l'action est encore plus affirmée dans les situations de fluidité politique, d'affaiblissement des référents sectoriels habituels (Havel parle "d'hypertrophie d'autodéfinition"44). En ce sens on peut dire, avec M. Dobry, que ces coups, ces mouvements tactiques qui scandent la dynamique des transitions, sont des "technologies rudimentaires d'objectivation" des identités et des groupes sociaux. Mais en même temps ces affirmations d'identité sont rendues plus difficiles et incertaines par les propriétés du contexte d'action. Dans un contexte d'interdépendance "élargie", les conséquences, la signification des coups échappent en grande partie aux acteurs qui doivent ensuite s'aligner sur l'interprétation qui en est faite ou dépenser beaucoup d'énergie pour la modifier. Le désarroi qu'expriment les intellectuels tchèques en 1990, les questions qu'ils se posent quant à leur position après leur engagement dans l'action politique en témoigne.

Ces propriétés de "l'interdépendance tactique élargie" s'observent encore plus dans la compétition que se livrent les acteurs des transitions pour la définition de la réalité. On sait sur le long terme, le rôle que joue l'imposition d'une vision légitime de la société dans la construction et la stabilisation sociale de la réalité. Conjoncturellement, la violence symbolique importe aussi. Dans les situations de transition, les luttes symboliques pour la définition légitime des enjeux sont directement des luttes pour le pouvoir qui "façonnent l'arène politique" (Collier). L'influence du contexte de fluidité y est manifeste. D'abord, les "définitions" qui émergent et qui structurent les perceptions sont directement en rapport avec les coups tactiques des parties. Elles naissent de l'interaction, de l'activité tactique des protagonistes du conflit. Empiriquement, il s'agit d'analyser les "processus par lesquels s'élaborent, se négocient et émergent (...) des définitions des situations qui présentent pour chacun d'eux des avantages inégaux" 45 . Ce programme de recherche éclairerait l'analyse des transitions. Ensuite, les "définitions" concurrentes de la "réalité" sont interdépendantes et subissent l'attraction conjoncturelle des "saillances", qui servent de point de convergence des interprétations, des anticipations. C'est en fonction de ces "points focaux" que va être déchiffrée la situation, que vont s'établir les estimations et que peuvent se cristalliser les règles du jeu.

Le changement de régime en Pologne illustre ce jeu des saillances, ce poids du contexte de fluidité dans la définition des situations et dans la dynamique des transitions. Après les discussions de la Table Ronde de février 1989, la situation était claire, bien définie entre les acteurs : instauration du pluralisme syndical, promesse de légaliser Solidarité, restauration de la liberté d'association et d'opinion (média), mise en place d'un système parlementaire à deux chambres et d'un Président de la République doté de pouvoirs étendus, élu par le Parlement. Ainsi établies, les nouvelles règles devaient assurer la domination du POUP sur le régime de transition ${ }^{46}$ (jusqu'aux élections libres prévues pour 1993). L'opposition n'aurait droit qu'à $35 \%$ des sièges à la Diète. Mais les élections du 5 juin 1989 (où Solidarité emporte la totalité des 161 sièges qui lui étaient accordés à la Diète et $99 \%$ des sièges au Sénat) remettent en cause le scénario défini par le pacte de la Table Ronde. Le jeu, "sectorisé", normalisé, par cet accord entre dans une nouvelle 
dynamique conflictuelle : l'été 1989 connaît un regain de mobilisation multisectorielle, une intensification de la fluidité politique. Les règles du jeu acceptées par Solidarité six mois plus tôt sont annulées et la situation se redéfinit au fil des coups. Dans ce contexte d'incertitude, de quasi-double pouvoir, l'apostrophe d'A. Michnick ("A Vous le Président, à Nous le Premier ministre") cristallise les interprétations. Les calculs, les anticipations des divers acteurs sont "pris" par cette interprétation qui s'impose comme seule légitime. Désormais, c'est autour de cette "saillance", de cette solution focale que se redéfinit la situation, que se structurent les nouveaux rapports de force, que se façonne le nouveau régime ${ }^{47}$.

L'exemple polonais témoigne ainsi de la fluctuation des ressources et de la mobilité des enjeux dans les conjonctures fluides. Il montre comment au fil des coups et des événements la structure du jeu se modifie, les perceptions et les anticipations sont réévaluées en fonction d'une part de cette variation des ressources, d'autre part des "saillances" qui émergent du conflit. Il traduit au fond le poids du contexte de fluidité politique sur le comportement des acteurs dans la dynamique des transitions.

\section{Problèmes de gestion, de légitimation et de consolidation démocratiques}

Il semble possible d'affirmer que "ce sont les propriétés des contextes d'interdépendance élargie qui commandent l'intelligibilité d'un grand nombre de "problèmes" ou de "dilemmes" qui s'imposent dans les conjonctures critiques aux acteurs sociaux"48. Conformément à la double hypothèse de départ, c'est dans ces propriétés contextuelles de la fluidité politique, dans les changements structuraux que nous avons cherché la source de l'incertitude des processus de gestion et de consolidation des démocraties naisssantes. C'est ainsi que peuvent s'interpréter les problèmes de gouvernabilité des transitions. La difficile maîtrise des réformes par exemple, la périlleuse élaboration des "programmes de légitimation démocratique" (Hermet), des "agendas initiaux" (Linz), peuvent se comprendre au regard de l'effondrement des critères routiniers d'évaluation (dû à la perte d'emprise des logiques sectorielles), au regard de la mobilité des enjeux et des préférences, de la fluctuation des ressources et du jeu des "saillances". Comment le contexte de fluidité "pèse"-t-il sur cette gouvernabilité et, inversement, comment l'action des dirigeants peut-elle contribuer à réduire cette fluidité politique? Dans quelle mesure par leurs efforts pour contrôler les processus de transition, et en maitriser l'incertitude parviennent-ils "non seulement à négocier tel ou tel virage, mais à orienter cette dynamique sur une trajectoire de long terme" ${ }^{149}$, autrement dit à légitimer et consolider le nouveau régime? Telles sont les questions qui peuvent guider une réflexion sur les agendas, les réformes de transition qui voudrait combiner analyse stratégique et intérêt pour les "structures" dans les contextes de fluidité des transitions. C'est ainsi que l'on peut tenter d'interpréter les dilemmes "linziens" de la légitimation démocratique, les problèmes de réforme économique "by democratic design" (C. offe), de gestion de la menace militaire ou l'échec de certaines expériences (celles du Vénézuela du Trieno ou Chili d'Allende par exemple) ${ }^{50}$. On observe dans cette aléatoire gestion des transitions, le poids et les propriétés du contexte de fluidité.

Mais les acteurs ne font pas que subir les logiques d'action de ce contexte. Ils s'efforcent de les maîtriser par divers mécanismes. Ainsi au Bénin, quand le Président 
Kérékou sous la pression de la rue et des bailleurs de fonds annonce (décembre 1989) la tenue d'une "Conférence des Forces Vives de la Nation", il cherche à "resectoriser" le jeu politique. Mais la Conférence, conçue comme un cadre d'expression des doléances, un moyen de gérer la fluidité de la crise, échappe à son instigateur. Au fil des coups, dans un contexte de forte tension, de mobilisations multisectorielles, les rapports de force et les perceptions se modifient. On est dans un contexte de forte désobjectivation où, selon les mots d'un observateur, règne un "climat de gravité, d'angoisse, le sentiment d'une issue imprévisible qui échappe aux prises de la collectivité" ${ }^{51}$, où les esprits, les forces de l'invisible sont convoqués pour faire face à l'effondrement des repères routiniers, à la perte d'emprise des logiques sectorielles. Dans ce cadre, la lutte pour la définition de la situation est âpre : alors que Kérékou essaie d'accréditer sa vision d'une Conférence-Palabre, d'une simple assemblée consultative (comme il l'avait expérimenté en 1979), un représentant de l'opposition (A. Hountoundji) proclame la souveraineté de la conférence nationale et la suprématie de ses décisions. Ce "speech act", ce "coup" s'impose comme saillance, cristallise les débats, les interprétations et "configure l'arène politique" (Collier). Dans leur lutte pour le pouvoir, les acteurs de la Conférence ont ainsi contribué à "resectoriser" le jeu mais dans un sens opposé aux intentions initiales de Kérékou et dans des proportions plus importantes (en s'autoproclamant représentants des groupes mobilisés, les délégués à la Conférence ont canalisé les mobilisations contestataires). Le nouveau Président vénézuélien, Romulo Betancourt, dans son premier discours de 1958, cherche aussi à limiter les effets de la "désectorisation de l'espace social". Face aux manifestations multisectorielles qui se poursuivent, il affirme que "le peuple dans l'abstait n'existe pas. Le peuple, c'est les partis politiques, les syndicats, les secteurs économiques organisés, les organisations professionnelles, les groupes universitaires..." ${ }^{52}$. En affirmant l'autonomie du politique et la nécessité d'organisation, de représentation institutionnelle des intérêts, il tente de replacer le jeu politique dans son arène légitime, son cadre normal, routinier. En insistant pour que la politique soit laissée aux professionnels, aux partis, pour que l'expression des intérêts soit confiée aux syndicats, aux groupes organisés, il lutte contre la "désectorisation" sociale. Il appelle à la reconnaissance de la division sectorielle de la société, à la reconnaissance de l'autonomie de chaque secteur, au retour à ce que l'on pourrait appeler le "paradoxe de la normalité"53.

D'autres mécanismes peuvent contribuer à réduire la fluidité politique des transitions, à gérer l'incertitude. La rédaction d'une constitution, la mise en place de nouvelles institutions, la codification de nouvelles règles sont quelques uns de ces procédés de "l'invention démocratique" ${ }^{54}$. L'annonce, l'organisation d'un scrutin peut aussi avoir pour effet de "resectoriser" le jeu politique, d'atténuer les traits de la fluidité (canalisation des griefs, stabilisation et évaluation des ressources, fixation des règles, identification des acteurs clés...) ${ }^{55}$. La conclusion d'un pacte (Pacte de Punto Fijo au Vénézuéla, Pactes de la Moncloa en Espagne, Tables Rondes dans les pays de l'Est...) peut également se lire ainsi : à court terme il contribue par la fixation de règles, de repères, de garanties, par la canalisation des revendications à réduire la fluidité. A moyen et long terme, les "transactions collusives" qu'il établit ou rétablit, les reconnaissances qu'il institue, peuvent être le signe du retour à un jeu sectorisé et à sa consolidation ${ }^{56}$. Aborder les pactes de transition sous l'angle des "transactions collusives" et des caractères structurels de la fluidité politique peut être fécond. Réducteurs de cette fluidité par leurs effets de court et moyen terme, ils en sont aussi révélateurs. A travers les propriétés des pactes, on discerne les traits propres aux 
conjonctures fluides; fluidité dont on observe aussi le poids dans les conditions de possibilité et de stabilité des pactes ${ }^{57}$.

C'est aussi dans cette perspective, c'est à dire au regard des traits structurels de la fluidité, que l'on peut comprendre et interpréter les modalités concrètes de la consolidation démocratique : "sectorisation" de l'espace sociopolitique, rétablissement des "transactions collusives" entre champs sociaux... L. Morlino décrit la consolidation comme un double mouvement : (a) un processus de fixation, d'objectivation de normes (démocratiques) de comportement partagées par la majorité des acteurs; (b) un processus de mise en oeuvre des institutions et de mise en place de nouveaux circuits politiques qui structurent les intérêts et assurent la médiation entre la société et l'Etat ou entre les divers secteurs sociaux ${ }^{58}$. Empiriquement, décrire la consolidation démocratique au concret c'est donc voir comment se resectorise l'espace social, comment se constituent de nouveaux réseaux d'interaction entre acteurs et champs sociaux, comment se routinisent entre eux des relations et comment s'objectivent ces rapports. Deux pistes de recherche s'ouvrent alors : Analyser comment s'établissent les relations entre les nouveaux gouvernants et les groupes socialement dominants (ou qui émergent comme socialement dominants - par exemple les nouvelles bourgeoisies à l'Est). Ce qui est en jeu ici, c'est la formation de "nouvelles élites" (ou la reconversion sous d'autres formes des mêmes élites), la consolidation de transactions collusives entre les acteurs dominants des divers secteurs sociaux. La question est importante en Afrique où s'observe particulièrement le chevauchement (straddling) des positions de pouvoir politique et économique ${ }^{59}$ : les transitions ne sont-elles qu'un avatar du "processus d'assimilation réciproque des élites" (Bayart) à l'oeuvre depuis les indépendances ou traduisent-elles l'émergence de nouveaux segments sociaux? Analyser comment s'établissent les relations entre dirigeants politiques et dirigeants d'organisations représentant les divers intérêts professionnels. Ce qui est en jeu ici, c'est la cristallisation et la consolidation de certaines formes d'intermédiation des intérêts, ce sont les transactions collusives de "l'Etat en action", de "l'Etat au concret" qui peuvent contribuer à la régulation et à la légitimation structurelle des nouveaux régimes ${ }^{60}$.

Penser les transitions au regard des propriétés de la fluidité peut donc aider à penser à la fois les problèmes tactiques et stratégiques des transitions mais aussi les processus de plus long terme de construction démocratique.

Mais introduire, avec la consolidation démocratique, la variable temporelle prouve aussi la nécessité d'affiner le modèle de la fluidité politique pour l'appliquer aux transitions. Les processus de démocratisation diffèrent en effet, au moins sur un point, des crises multisectorielles dont M. Dobry trace les traits : ils ne s'inscrivent pas dans la même durée. Les crises "classiques" sont des phénomènes somme toute conjoncturels par rapport à la dynamique longue et évolutive des transitions. Etalées dans le temps, celles-ci ne sont pas à tout moment et de façon uniforme des situations de fluidité politique. Le jeu est plus ou moins "sectorisé" selon les moments. Il faut insister sur cette nuance importante et tenter de préciser le dégré de fluidité des transitions en fonction des moments, des séquences temporelles.

Les processus de transition diffèrent aussi dans leurs modalités. Peut-on distinguer selon les types, les modalités de transition, des configurations et des degrés différents de fluidité politique? Un grand nombre de typologies ont été proposées en fonction des rythmes de démocratisation, des acteurs engagés, des stratégies et des ressources 
employées ${ }^{61}$. On peut alors émettre l'hypothèse que la fluidité politique d'une transition sera plus ou moins élevée selon qu'elle s'opère de façon graduelle ou brutale, par "transaction" ou par "rupture", qu'elle est "imposée", "octroyée" ou "pactée"... Mais c'est oublier que de tels classement "essentialisent" des trajectoires, objectivent des différences qui, souvent, ne sont que de circonstance et évoluent au fil de chaque transition. Le Portugal, par exemple, a engagé sa transition avec le coup d'Etat d'officiers subalternes dans un contexte de défaite extérieure imminente. Mais leur prise de pouvoir a été suivie d'une "réaction des masses", d'une intense mobilisation multisectorielle qui a d'abord orienté le processus dans une direction réformiste puis vers la voie révolutionnaire. Après l'échec de celle-ci, "le Portugal est resté quelques temps suspendu entre les réformes et la transition imposée" ${ }^{62}$. A première vue - la notion de fluidité étant associée à celle de crise - il semble évident qu'une transition douce, "pactée", sera moins fluide qu'un changement brutal imposé par la force. C'est pourtant loin d'être évident. Une transition, estime Juan Linz, est toujours un processus complexe combinant réforme et rupture ${ }^{63}$. Une transition négociée, comme le montrent Rustow et Przeworski, est souvent le résultat d'un conflit prolongé entre acteurs ayant épuisé toutes leurs ressources dans un conflit multisectoriel sans pouvoir imposer aux autres leur définition de la situation. Les traits de la fluidité, de la désectorisation de l'espace social peuvent alors s'observer dans les négociations (mobilité des enjeux, dispersion des marchandages...), dans les pactes voire dans les solutions institutionnelles. A l'inverse, un changement imposé d'en haut, par la force (un coup d'Etat par exemple), ne traduit pas forcément une situation de mobilisation multisectorielle, une large désectorisation du jeu politique.

Celle-ci peut se manifester dans tous les types de transitions. La fluidité politique ne semble pas mécaniquement liée aux mode de transition, elle peut émerger, conjoncturellement dans des situations diverses sous l'effet de mobilisations multisectorielles. Au fond, les diverses typologies masquent peut-être la similarité des rapports sociaux, des configurations "structurelles" propres aux situations de changement. Il parait alors impossible de distinguer, selon les types de transition, des différences de nature dans leur fluidité. On peut seulement relever des différences de degré, d'intensité, selon les moments. Il s'agit alors de préciser cette fluidité inégale des transitions en introduisant la variable temporelle. L'opération n'est pas sans danger. Périodiser - c'est à dire au sens strict repérer dans le temps des configurations stables de variables - des processus où la plupart des "paramètres sont en flux est déjà difficile. Ensuite, distinguer des étapes, des séquences, comporte un biais finaliste : "l'illusion de l'histoire naturelle" (Dobry) qui ferait croire à une régularité, une linéarité de la démocratisation pourtant largement aléatoire. "Mesurer" ou observer les divers degrés de fluidité des transitions suppose alors de s'intéresser de plus près aux processus de mobilisation multisectorielle, à leurs émergences, leurs rapports au changement politique. C'est, à nos yeux, à cette condition que l'on pourra avancer dans la compréhension des processus incertains de démocratisation et affiner le modèle de la fluidité politique des transitions.

Mobilisations collectives et fluidité politique des transitions 
31 Penser les transitions démocratiques comme des situations de fluidité politique inégale (et préciser empiriquement ces degrés divers de fluidité) c'est donc s'intéresser au "comment", au contexte de transition lui-même et non plus seulement au "pourquoi" des changements de régime, c'est tenter de comprendre ce qui s'y joue concrètement. Cela signifie centrer son attention sur les transformations d'état que subissent les structures sociales dans les conjonctures critiques sous l'effet des mobilisations "multisectorielles". C'est à dire chercher l'incertitude des transitions dans ces changements structuraux (la "désectorisation de l'espace social", la "crise des transactions collusives", la "désobjectivation" corrélative de l'ordre sociopolitique) qui définissent au niveau micropolitique des logiques d'action originales ("l'interdépendance tactique élargie" et ses propriétés dérivées) et marquent les problèmes de gestion, de légitimation, de consolidation démocratique. Aborder les processus de transition sous l'angle des propriétés de la fluidité politique, amène ainsi à réflechir plus précisément aux rapports qui se nouent entre mobilisations collectives et changement politique.

Malgré son importance empirique (et à nos yeux théorique) le problème des mobilisations collectives en période de transition est resté négligé à la fois par les diverses approches des transitions, les travaux sur l'action collective et par les "area studies". Parmi ces dernières, peu d'études empiriques ont été consacrées aux manifestations, aux mouvements de revendication démocratique qui, pourtant, ont marqué nombre de pays de l'Est et du Sud depuis 1989. Souvent pessimistes quant aux perspectives de mobilisation et de contestation ouverte, les études est-européennes par exemple ont longtemps oscillé entre l'analyse des formes cachées de dissidence ${ }^{64}$ et le constat d'apathie ${ }^{65}$. Le développement de Solidarité, a certes ouvert la voie à l'étude des mouvements sociaux ${ }^{66}$. Mais les événements de 1989 ont suscité plus d'observations sur les formes de renaissance de la "société civile"67 que de véritables analyses concrètes des mobilisations collectives et de leur rapport au changement politique. De même, les recherches africanistes qui ont pourtant montré l'étendue de la "revanche des sociétés africaines" (Bayart), la diversité des pratiques de contestation de l'Etat post-colonial notamment les travaux consacré au "politique par le bas" et au "disengagement"68 - ont encore peu étudié ces récents mouvements de protestation ouverte qui, de Lomé à Libreville, de Bamenda à Cotonou ou Lagos, ont scandé les évolutions politiques depuis $1989^{69}$. De leur côté, les récentes théories des transitions, comme nous l'avons vu, ont réhabilité les variables micropolitiques en se focalisant sur le rôle stratégique des élites. Les mouvements sociaux, les mobilisations collectives, dans ce cadre élitiste, sont rarement abordés en tant que tels, et quand ils le sont, c'est en termes de "masses" ( $\mathrm{T}$. L. Karl) ou de "résurrection de la société civile" (O'Donnel et Schmitter). Plus conceptuelles qu'empiriques, ces analyses visent plus à modéliser le rôle des mobilisations dans l'effondrement des régimes qu'à rendre compte de leur réalité complexe et de leur rapport au contexte de fluidité des transitions. Symétriquement, les diverses sociologies de l'action collective, ne se sont guère intéressées aux transitions et aux mouvements de revendication démocratique. A notre connaissance, aucune des écoles - de la psychologie des foules, de la frustration relative ${ }^{70}$, de la mobilisation des ressources (courant économiste ${ }^{71}$ ou courant politique ${ }^{72}$ ), des nouveaux mouvements sociaux ou de l'identité ${ }^{73}$ - n'a posé ouvertement le problème des mobilisations collectives en période de transition. Les différents modèles et hypothèses, élaborés sur le terrain occidental, n'ont pas encore été confrontés - à de 
rares exceptions près $^{74}$ - aux mouvements de contestation et de revendication démocratique de l'Est et du Sud. Pour S. Tarrow ${ }^{75}$, les schémas disponibles actuellement seraient insuffisants pour penser ces vagues de mobilisation et leur rôle dans les changements de régime. La contrainte des institutions étant sous-évaluée, il faut selon lui, recourir à de nouvelles variables (touchant à la "structure des opportunités politiques") pour interpréter ces nouveaux "cycles de protestation et dépasser le paradoxe olsonien de l'action collective, particulièrement pesant en situation autoritaire. C'est également l'avis de C. Tilly qui estime que les événements des années 1989-90 devraient susciter de nouvelles réflexions sur les processus de mobilisation ${ }^{76}$.

Penser les transitions comme des situations de fluidité politique - où la "dynamique des mobilisations multisectorielles" pèse à la fois sur les "structures" sociales et les comportements- pourrait y aider. Au delà des effets de ces mobilisations de crise ("désectorisation" et propriétés dérivées...), il faut alors analyser ces processus de mobilisation collective de transition en eux-mêmes, approfondir empiriquement leur connaissance pour mieux comprendre leur portée et leur rapport au changement politique.

En guise de conclusion, quelques pistes de recherche peuvent être esquissées en ce sens. Comment naissent les motivations et comment passe-t-on des motivations à l'action? Ces questions classiques de la sociologie des mobilisations sont d'autant plus importantes qu'elles se posent dans des contextes de remise en cause des régimes autoritaires. Comment expliquer en effet qu'en 1989-90, en Afrique, les individus dépassent le registre de la ruse, de l'escapade, descendent dans la rue et choisissent la voie de l'action collective alors qu'il semblait acquis que les modes populaires d'action politique ne puissent "déboucher sur des mouvements sociaux durables"77. Comment expliquer le passage d'un comportement de résistance, de dissidence passive à une protestation ouverte? A. Hirschman observe que dans les pays de l'Est la relation entre les deux attitudes était inverse jusqu'en 1988-1989. La possibilité d'exit (réelle ou symbolique) réduisait les probabilités de "voice"78. Les hypothèses de l'école de la mobilisation des ressources, supposant une rationalité coûts/bénéfices et l'existence de groupes fortement structurés, ne semblent pas suffisantes pour expliquer cette mutation des comportements et dépasser le paradoxe olsonien de l'action collective, particulièrement pesant à l'Est ou en Afrique. Faut-il rechercher alors, avec les théories de l'identité (Melucci, Pizzorno), le sens de l'action dans l'action elle-même plutôt que dans les buts poursuivis? Ou comme le suggère Tarrow, rapporter le problème de la rationalité de l'engagement aux variations de la "structure d'opportunité politique", articuler l'action collective au contexte et à la dynamique de transition, aux propriétés de la fluidité politique des transitions (notamment aux phénomènes de désobjectivation liés à la crise des transaction collusives)? Conformément aux hypothèses de départ, c'est dans cette voie qu'il semble possible d'analyser le passage de la résistance passive à la protestation publique, des motivations à l'action en période de transition.

Comment passe-t-on ensuite de l'engagement individuel à l'action collective? Autrement dit, comment se mobilise-t-on dans ces conjonctures fluides? M. Kaase rappelait récemment l'importance des organisations prenant en charge les griefs, que ce soient les "communautés pré-existantes" d'Obershall, les "entrepreneurs de mouvement" de Zald et Mac Carthy ou les "espaces d'intégration" de Melucci. S'agit-il de mobilisations organisées, dirigées par des groupes structurés d'activistes ou de 
mouvements de masse plus ou moins spontanés? Est-on en présence d'acteurs déjà constitués, de groupes qui se constituent en acteurs collectifs par leur participation aux conflits $^{79}$, de structures de coordination pré-existantes, stables ou contingentes, élaborées à la hâte pendant la crise ? Au Bénin, on l'a vu, alors que s'érodent les structures officielles de mobilisation de masse, des organisations parallèles et des réseaux se mettent en place. Il s'agit alors d'identifier ces "organisations de mouvement" (SMO) de reconstituer ces structures de coordination. Cela permettrait d'évaluer concrètement leur rôle dans la contestation et dans l'émergence d'un contexte de fluidité politique. Cela pourrait aussi ouvrir de nouvelles pistes dans l'étude des mobilisations collectives. Etant données, dans la plupart des situations, l'absence de véritables "organisations de mouvements" anciennement structurés et la fluidité du contexte de transition, ne faudra-t-il pas suivre les récentes propositions de Tilly et conceptualiser les acteurs des mobilisations de 1989-1992 au Bénin, au Togo ou dans les pays de l'Est comme des "réseaux sociaux changeants, contingents et construits" 80 ? Cela irait contre l'hypothèse classique de continuité organisationnelle des mouvements sociaux, "pré-condition" des mobilisations pour de nombreuses théories. Cette hypothèse de plasticité, de fluidité, si elle se confirmait constituerait une des originalités des mobillisations de transition et pourrait générer de nouvelles conceptualisations de l'action collective en période de crise.

Les problèmes de gestion du sens des mobilisations sont également importants dans la dynamique des transitions. Les luttes symboliques autour des manifestations sont toujours très importantes ${ }^{81}$. Elles s'avèrent cruciales dans le contexte de fluidité, de désobjectivation" des périodes de transition où la lutte pour l'imposition du sens est aussi une lutte pour le pouvoir. Partant de l'hypothèse qu'en situation de crise les "définitions" qui émergent sont le produit de l'activité tactique, conflictuelle, des différentes parties, il s'agit d'analyser les processus, les interactions au cours desquels s'élaborent, se négocient dans la mobilisation des "définitions" de la situation. Comment les acteurs de l'opposition peuvent-ils imposer dans la lutte contre le pouvoir et par-delà la diversité des revendications, l'image d'un mouvement de masse pour la démocratie? La convergence des interprétations autour de la solution pluraliste ne peut-elle se comprendre, dans le contexte de fluidité et de lutte, par l'attraction des "points focaux"? Les propriétés de la fluidité politique pourraient permettre de comprendre comment dans l'interaction stratégique, au fil des coups entre les groupes mobilisés et le pouvoir, le multipartisme, la "démocratie" peuvent s'imposer comme une "saillance situationnelle" qui structure les perceptions et les interprétations.

Préciser ces logiques de mobilisation collective, leur rapport au contexte de fluidité politique, pourrait ainsi faire progresser la compréhension des processus de démocratisation, des dynamiques de transition. Cela suppose et nécessite d'amples investigations empiriques. 


\section{NOTES}

1. La notion de "transition démocratique" a fait l'objet de longues discussions. Utilisée d'abord pour désigner le changement pacifique de régime en Espagne, elle a fait ensuite l'objet de nombreux débats. Rejetée pour ses connotations et surtout son finalisme qui néglige l'incertitude et la réversibilité des processus de démocratisation, elle est, dans les années 1990, de plus en plus remise en question (voir le dernier rapport de la Banque Mondiale) et remplacée par la notion de réforme qui renvoit plus aux problèmes de changement systémique, de gestion économique et de "good governance". Sans entrer dans ces débats terminologiques, on peut ici retenir l'expression de façon pratique et minimaliste : dans son acception temporelle, la transition démocratique est simplement la période ambigüe de changement de régime. On peut la définir comme une période de flux institutionnel, d'incertitude, où la norme démocratique tend à s'imposer comme critère de légitimation et oriente les perceptions et les comportements de la majorité des acteurs.

2. Voir Almond (G.), Verba (S.), The Civic culture. Princeton, Princeton Univ. Press, 1963, 562 p. Lerner (D.), Pevsner (L.), The Passing of traditional society, New York, Free Press, 1958, 466 p. Eisenstadt (S. N.), "Social change, differenciation and evolution", American Sociological Review, n²9, juin 1964, pp. 375-387. Moore (B.), Les Origines sociales de la dictature et de la démocratie, Paris, Maspéro, 1969, 431 p. Lipset (S. M.), "Some social requisites of democracy : economic development and political legitimacy", American Political Science Review, 53 (1) mars 1959, pp. 69-105, ainsi que l'Homme et la Politique, Paris, Le Seuil, 1960.

3. Pour une synthèse et une mise en perspective critique de ces approches, voir Hermet (G.), Sociologie de la construction démocratique, Paris, Economica, 1986.172 p.

4. Voir Rustow (D.), "Transition to democracy : toward a dynamic model", Comparative Politics, (2), 3 avril 1970, pp. 337-363.

5. Voir Huntington (S.), The Third Wave : democratization in the late twentieth century, Norman, University of Oklahoma Press, 1991, 366 p.

6. Si ces réorientations de paradigme peuvent s'interpréter en termes "khuniens" comme une réponse à des "anomalies", à des contre-exemples, on peut penser qu'elles ont aussi triomphé pour les besoins de la cause. La réévaluation de l'action politique (jusqu'au "volontarisme" de certaines analyses) nourrit plus efficacement l'optimisme démocratique que l'attente passive d'une hypothétique réalisation des préconditions du pluralisme. Le poids de "l'Histoire réelle" apparaît ici directement. Engagés dans l'action politique, certains chercheurs ont ainsi changé leurs orientations théoriques, non sans problèmes méthodologiques.

7. Voir par exemple l'interprétation que fait A. Stepan de la transition brésilienne. Certes des facteurs structurels ont affaibli le régime militaire du président Geisel, mais les raisons de sa chute sont à chercher ailleurs. Dans ses contradictions politiques internes, dans la volonté d'une faction de l'armée de trouver dans la société civile des alliés pour contrer l'influence croisssante des services de renseignement (le fameux SNI) au sein de l'Etat. Voir Stepan (A.), Rethinking military politics. Brazil and the Southern Cone, Princeton, Princeton Univ. Press, 1988, 172 p. 
8. Voir O'donnell (G.), Schmitter (P.), Transitions from authoritarian rule. Tentative conclusions about uncertain democracies, Baltimore, The J. Hopkins Univ. Press, 1986. Przeworski (A.), Democracy and the market, Cambridge, Cambridge Univ. Press, 1991.

9. Rustow (D.), art. cit., p. 353.

10. Observons, contre la thèse de l'accord sur les valeurs précondition de la démocratie, que le consensus, l'accord sur les règles (voire les fins) est ici moins le prérequis que le résultat du processus dynamique de démocratisation (la consolidation est ici vue comme processus de socialisation civique)

11. Voir Linz (J. J.), The Breakdown of democratic regimes, Baltimore, The J. Hopkins Univ. Press, 1978, (vol. 1), 125 p.

12. Di Palma (G.), To Craft democracies. An essay on democratic transitions, Berkeley, Univ. of California Press, 1990, p. 8.

13. Voir Przeworski (A.), "Democracy as a contingent outcome of conflict" in Elster (J.), Slagstad (R.), eds, Constitutionnalism and democracy. Cambridge, Cambridge Univ. Press, 1987, pp. 59-80.

14. Hermet (G.), Aux frontières de la démocratie, Paris, PUF, 1983, p. 207.

15. Voir Hirschman (A.), "Models of reform mongering" in Journeys toward Progress, Garden City, Doubleday, 1965 ; Przeworski (A.), Democracy and the market, op. cit. et O'donnell (G.), "Notes for the study of democratic consolidation in contemporary Latin America" à paraître dans Delegative democracy.

16. Il serait trop long de détailler ici ces approches. Pour une mise en pespective précise, voir Collier (D.), Norden (D.), "Strategic choice models of political change in Latin America" in Comparative Politics, 24 (2), janvier 1992, pp. 229-243.

17. Voir Schelling (T.), Stratégie du Conflit, Paris, PUF, 1986, 312 p.

18. C. Offe explique ces impasses par la confusion des niveaux d'analyses. Comment rendre compte microsociologiquement de changements qui n'affectent pas seulement les règles d'intéraction politique mais également la société dans ses structures profondes ? c'est le "dilemme de la simultanéité", de la "triple transition" que ne peut résoudre le paradigme du choix rationnel. Voir Offe (C.), "Capitalism by democratic design. Democratic theory facing the triple transition in Eastern Europe" in Social Research, 58 (4) 1991, pp. 865-902, traduit dans la RFSP, 42 (6) décembre 1992, pp. 923-942.

19. Voir Karl (T. L.), "Dilemmas of democratization in Latin America", Comparative Politics, 23 (1), oct. 1990, pp. 1-21 et Karl (T. L.), Schmitter (P.), "Les modes de transition en Amérique latine, en Europe du Sud et de l'Est", Revue Internationale de Sciences Sociales, $\mathrm{n}^{\circ} 128$, mai 1991, pp. 285-301.

20. Voir Collier (D.), Berins-Collier (R.), Shaping the Political Arena. Critical junctures, the labor movement and the regime dynamics in Latin America, Princeton, Princeton Univ. Press, 1991.

21. Almond (G.), Flanagan (S.), Mundt (R.), eds, Crisis, Choice and Change, Boston, Little Brown, 1973, p. 67.

22. Voir Dobry (M.), Sociologie des crises politiques. La dynamique des mobilisations multisectorielles, Paris, Presses de la FNSP, 1986.

23. Voir Almond (G.), Flanagan (S.), Mundt (R.), eds, op. cit., notamment Flanagan (S.), "Models and methods of analysis", pp. 43-102.

24. Dobry (M.), op. cit., p. 138.

25. La complexité structurelle des sociétés désigne une évidence sociologique : la multiplicité des sphères sociales, des champs, des secteurs sociaux différenciés et 
institutionnalisés qui, par leurs stratégies de distinction et leurs relations enchevêtrées, dessinent la trame du "tissu social".

26. Dobry (M.), op. cit., p. 141.

27. Ibid. p. 141.

28. Le postulat sous-jacent à cette propriété touche à la rationalité de l'action sociale : dans les conjonctures routinières, les anticipations, les évaluations et plus généralement l'activité tactique des acteurs s'effectuent non pas selon une rationalité parfaite et universelle, mais en fonction des enjeux, des règles du jeu ("normatives" ou "pragmatiques"), des types de ressources propres à chaque secteur ou champ social. Les acteurs sont pris dans des "toiles de signification" (C. Geertz), des "zones limitées d'interdépendance tactique" (M. Dobry) qui fournissent les critères, repères et instruments de calcul, d'évaluation et d'interprétation des situations.

29. Voir Dobry (M.), op. cit., pp. 154-158 et pp. 262-287.

30. Allen (C.), "Restructuring an authoritarian State. Democratic renewal in Bénin", Review of African Political Economy, 54, 1992, p. 47.

31. Ibid. p.48.

32. Staniszkis (J.), Pologne : la Révolution autolimitée, Paris, PUF, 1982, p. 117.

33. Ibid, p. 134.

34. Sur cet aspect de la "normalisation" en Tchécoslovaquie après le printemps de Prague, voir le témoignage intime (au sens des "tyrannies de l'intimité"de R. Sennet) de Simecka (M.), Le rétablissement de l'ordre, Paris, Maspéro, 1979.

35. Voir les descriptions de T.G. Ash, La chaudière. Europe Centrale 1980-1990, Paris, Gallimard, 1990, p. 385 et suiv.

36. Voir Dobry (M.), op. cit., pp. 150-153.

37. Ibid. p. 159-160.

38. Sur ces stratégies de reconversion, voir $\operatorname{Mink}(\mathrm{G}$.), Lhomel (E.), "Conversion et adaptation des partis communistes" in Notes et Etudes documentaires, 4942 (43), 1991, pp. 33-47. Ainsi que Mink (G.), Szurek (J. F.), "Adaptation et stratégies de reconversion des anciennes élites communistes" in Mink (G.), Szurek (J. F.), op. cit., pp. 67-84.

39. Voir Hermet (G.), Les Désenchantements de la Liberté. La sortie des dictatures dans les années 1990, Paris, Fayard, 1993.

40. Dobry (M.), op. cit., p. 173.

41. Ibid. p. 161.

42. Ibid. p. 184.

43. Voir Pizzorno (A.), "Considérations sur les théories des mouvements sociaux". Politix, (9), 1990, pp.74-80 et Melucci (A.), "The new social movements : a theoretical approach" in Social Science Information, (19) 1980, pp.199-226. Pour une synthèse récente de ces théories de l'identité qui se proposent de dépasser le paradoxe olsonien de l'action collective, voir Martelli (D.), Identité et mobilisation, Paris, Univ. Paris I, 1992 (mémoire).

44. Entretien avec A. Michnick in Mink (G.), Szurek (J. C.), Cet étrange postcommunisme, Paris, La Découverte, 1992, p. 29.

45. Dobry (M.), op. cit., p. 198.

46. Voir Kaminski (B.), "Systemic underpinnings of the transition in Poland : the Shadow of the Round-Table agreement", Studies in Comparative Communism, 24 (2), juin 1991, pp. 173-190. Ainsi que Mink (G.), "Pologne. De la Table Ronde au double pouvoir" in L'URSS et l'Europe de l'Est, Paris, La Documentation Française, édition 1990, pp. 151-175. 
47. Rapporter ainsi l'établissement d'un jeu coopératif à l'attraction des "saillances", des points focaux peut aider à comprendre les phénomènes de convergence, les tendances à la coopération qui marquent les transitions pacifiques et que les analystes expliquent simplement par l'intérêt ou la "sagesse" des protagonistes.

48. Dobry (M.), op. cit., p. 173.

49. Hermet (G.), Aux frontières de la démocratie, op. cit., p. 207.

50. Nous nous permettons de renvoyer à notre travail (notamment au troisième chapitre et aux études de cas), Les Transitions démocratiques comme situations de fluidité politique. Gestion, Légitimation, Consolidation, Paris, IEP, 1992, (Mémoire de DEA sous la direction de M. Guy Hermet), 207 p.

51. Eboussi Boulaga (F.), Les Conférences Nationales en Afrique Noire, Paris, Karthala, 1993, p. 70.

52. Betancourt (R.), Tres anos de gobierno democràtico, Caracas, Imprenta Nacionàl, 1962, p. 245.

53. C'est à dire une situation normale, routinière où la stabilité sociale est assurée par les rapports, contradictoires, d'autonomie et d'interdépendance à la fois entre secteurs ou champs sociaux institutionnalisés.

54. Lefort (C.), L'invention démocratique, Paris, Fayard, 1981.

55. Sur les premières élections pluralistes, voir la Revue Française de Science Politique, vol. 43, nº, décembre 1993. Il faut ici remarquer que les effets politiques des premiers scrutins sur la dynamique des transitions (ou sur la cristallisation des systèmes de partis) ont été peu étudiés. Citons simplement les formalisation de O'donnell Et Schmitter, op. cit., notamment le chapitre VI, "Convoking elections (and provoking parties)", pp. 57-64, ou l'analyse plus empirique d'O. Dabene, "Elections et crise en Amérique centrale", Etudes, février 1990, pp. 159-172.

56. La littérature consacrée aux pactes est en revanche abondante. Depuis les travaux de Kirchheimer dans les années 1960 jusqu'aux analyses stratégiques de Przeworski ils ont été au centre de l'analyse des démocratisations, malgré le nombre somme toute limité de transitions "pactadas". Citons simplement les travaux de T. L. Karl, "Petroleum and Political pacts. The transition to democracy in Venezuela", Latin America Research Review, 22 (1), 1987, pp. 63-91 ; "Dilemmas of democratization in Latin America", Comparative Politics, 23 (1), 1990, pp. 1-20 et de P. Schmitter, "Patti e transizioni : mezzi non democratici a fini democratici ?", Rivista Italiana de Scienza Politica, 24 (3), 1984, pp. 363-382.

57. Nous développons cet argument dans notre mémoire, op. cit., pp. 96-114.

58. Voir Morlino (L.), "Consolidacion democràtica : definicion, modelos, hipòtesis", Revista Espanola De Investigaciones Sociologicas, $n^{\circ} 35,1986$, p. 272.

59. Voir Bayart (J. F.), L'Etat en Afrique. La politique du ventre, Paris, Fayard, 1989. 60. C'est l'hypothèse que l'on peut formuler à partir des travaux de Schmitter sur les rapports corporatisme/gouvernabilité ou les réflexions de Jobert et Muller sur la portée "positive" des interactions pouvoirs politiques/acteurs sociaux sur la régulation politique et la légitimité des régimes.

61. Voir par exemple, celle d'A. Stepan, "Path toward redemocratization : theoretical and comparative reconsiderations", in O'donnell (G.), Schmitter (P.), Whitehead (L.), eds, op. cit., (vol. 3), pp. 65-84, ou celle proposée par Karl (T. L.), Schmitter (P.), "Les modes de transition en Amérique latine, en Europe du Sud et de l'Est", art. cit.

62. Ibid., p. 296. 
63. Paradoxalement, c'est la formulation simultanée des deux positions par des adversaires aux ressources comparables qui rend possible l'ouverture politique. Voir Linz (J.), "Transition to democracy", The Washington Quarterly, été 1990, pp. 143-163. 64. Voir Havel (V.), Keane (J.), eds., The power of the powerless. Citizens against the State in central-eastern Europe, New York, Palach Press, 1985.

65. Voir Mason, Nelson, "Apathy and the birth of democracy". East European Politics and Society. 5 (2) 1991 ainsi que Marody (M.), "Perception of politics in polish society". Social Research. 57 (2) 1990. pp 257-274.

66. Voir Touraine (A.), Solidarité, Paris, Fayard, 1982

67. Voir Keane (J.), The redescovery of civil society, Londres, Verso, 1988 et Democracy and civil society. Londres, Verso, 1988. ainsi que Molnar (M.), La démocratie se lève à l'Est. Société civile et communisme en Europe de l'Est, Paris, PUF, 1990.

68. Pour une présentation (et une évolution), voir le récent recueil de Bayart (J. F.), Mbembe (A.), Toulabor (C.), Le politique par le bas. Contribution à une problématique de la démocratie en Afrique noire, Paris, Karthala, 1992. Pour un aperçu des travaux du courant anglo-saxon du "Disengagement" voir par exemple, Hyden (G.), Beyond Ujamaa in Tanzania. Underdevelopment and an uncaptured peasantry, London, Heineman, 1980 ; et pour une conceptualisation de ces relations "d'Incorporation/ Désengagement", voir Rothschild (D.), Chazan (N.), eds, The Precarious balance. State and civil society in Africa, Boulder, Westview Press, 1988.

69. A notre connaissance, seuls les travaux de M. Bratton et $\mathrm{N}$. Van de Walle posent ouvertement le problème des rapports entre mouvements de protestation et de réforme politique en Afrique noire depuis 1989. Voir Bratton (M.), Van De Walle (N.), "Popular protest and political reform in Africa" in Comparative Politics, 24(4) 1992, pp. 419-442 ; et dans une version remaniée, "Toward governance in Africa : popular demands and State responses", in Hyden (G.), Bratton (M.), eds., Governance and politics in Africa, London, L. Rienner, 1992, pp. 27-55.

70. Voir Gurr (T.), Why men rebel ?, Princeton, Princeton Univ. Press, 1970.

71. Voir Zald (M.), Mac Carthy (J.), Social movement in an organizational society, New Brunswick, Transaction, 1987.

72. Voir, Obershall (A.), Social conflict and social movements, Englewood, Prentice Hall, 1973 ; Tilly (C.), From mobilization to revolution, Englewood, Prentice Hall, 1978.

73. Voir, Offe (C.), "New social movements : challenging the boundaries of institutionnal politics", Social Research, 52, 1985, pp. 817-868.

74. Par exemple, Hirschman (A.), "Exit, Voice and the Fate of the GDR", World Politics, 45, 1993. pp.173-202., qui teste son modèle Exit/ Voice avec l'exemple de l'ex-RDA ; Wankel (C.), Anti-communist student organizations and the polish renewal, New York, St Martin Press, 1992, qui teste, lui, les hypothèses de la théorie de la mobilisation des ressources sur les mouvements étudiants polonais.

75. Voir Tarrow (S.), "Aiming at a moving target. Social science and the recent rebellions in Eastern Europe", P. S, 24 (1), 1991, pp. 12-20.

76. "Réclamer viva voce", Cultures et Conflits, ${ }^{\circ}{ }^{\circ}$, printemps 1992.

77. Bayart (J. F.), L'Etat en Afrique. op. cit., p. 223.

78. Voir Hirschman (A.), "Exit, Voice...", art. cit.

79. Sur ce processus d'objectivation des groupes pendant les mobilisations de transition, voir Grzybowski (C.), "Rural workers's movements and democratization in Brazil". Journal of Development Studies, 26 (4) 1990, pp. 19-43 ; ainsi que Le Roy (E.), 
"Mouvement paysan et transition démocratique dans le sud du Mali", L’Année Africaine, 1992-93, pp. 146-59.

80. Tilly (C.), "Réclamer viva voce", Cultures et Conflits, $n^{\circ} 5$, printemps 1992.

81. Voir Champagne (P.), "La manifestation comme action symbolique" in Favre (P.), La manifestation. 1990. pp. 329-356, et Dobry (M.) : "Calcul, concurrence et gestion du sens" in Favre (P.) : La Manifestation. pp.357-386.

\section{RÉSUMÉS}

Quels sont les processus qui conduisent à la "démocratisation" d'un régime ? Les modèles d'analyse et les approches classiques privilégient généralement la recherche des causes, l'établissement d'une "liste" de pré-conditions nécessaires pour que la transformation démocratique ait lieu. Toutefois, à vouloir systématiquement rechercher les causes, le pourquoi de ses transformations, on néglige les logiques propres des transitions démocratiques, périodes ambigües où la norme démocratique tend à s'imposer, les propriétés contextuelles, propriétés qui permettent de comprendre les problèmes de gestion des réformes, de légitimation, de consolidation démocratique ou les phénomènes de mobilisation collective. La compréhension de ces transitions passe donc par l'analyse de ses situations en terme de crises politiques, de fluidité politique.

INDEX

Mots-clés : démocratie, mobilisation

Index géographique: Afrique subsaharienne 